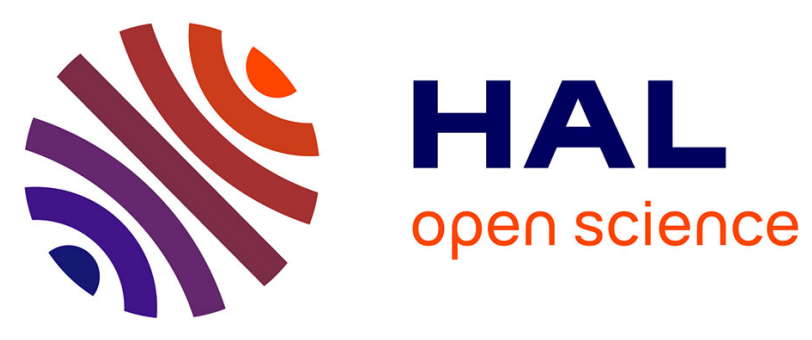

\title{
Enhanced Visual Analytics Services for the Optimal Planning of Renewable Energy Resources Installations
}

Votis Konstantinos, Yiannis Karras, Jörn Kohlhammer, Martin Steiger, Dimitrios Tzovaras, Elias Gounopoulos

\section{- To cite this version:}

Votis Konstantinos, Yiannis Karras, Jörn Kohlhammer, Martin Steiger, Dimitrios Tzovaras, et al.. Enhanced Visual Analytics Services for the Optimal Planning of Renewable Energy Resources Installations. 10th IFIP International Conference on Artificial Intelligence Applications and Innovations (AIAI), Sep 2014, Rhodes, Greece. pp.330-339, 10.1007/978-3-662-44722-2_35 . hal-01391060

\author{
HAL Id: hal-01391060 \\ https://hal.inria.fr/hal-01391060
}

Submitted on 2 Nov 2016

HAL is a multi-disciplinary open access archive for the deposit and dissemination of scientific research documents, whether they are published or not. The documents may come from teaching and research institutions in France or abroad, or from public or private research centers.
L'archive ouverte pluridisciplinaire HAL, est destinée au dépôt et à la diffusion de documents scientifiques de niveau recherche, publiés ou non, émanant des établissements d'enseignement et de recherche français ou étrangers, des laboratoires publics ou privés.

\section{(c)(1)}

Distributed under a Creative Commons Attribution| 4.0 International License 


\title{
Enhanced Visual Analytics Services for the Optimal Planning of Renewable Energy Resources Installations
}

\author{
Votis Konstantinos ${ }^{1}$, Yiannis Karras ${ }^{2}$, Jörn Kohlhammer ${ }^{3}$, Steiger Martin ${ }^{3}$, \\ Dimitrios Tzovaras ${ }^{1}$, Elias Gounopoulos ${ }^{4}$ \\ ${ }^{1}$ Information Technologies Institute/ Centre for Research and Technology Hellas \\ 57001, Thessaloniki, Greece \\ \{kvotis, dimitrios.tzovaras\}@iti.gr \\ ${ }^{2}$ Inaccess . \\ 15125, Athens, Greece \\ \{jkarras\}@inaccess.com \\ 3 "Fraunhofer IGD" \\ Darmstadt, Germany \\ \{joern.kohlhammer, Martin.Steiger\}@ igd.fraunhofer.de \\ ${ }^{3}$ Technological Educational Institute of Western Macedonia (TEIWM) \\ 51100, Grevena, Greece \\ \{elgounop\}@teikoz.gr
}

\begin{abstract}
This paper presents an integrated and novel service environment for real-time interactions between users, as well as enhanced visualization and decision support services over extremely large volumes of heterogeneous Renewable data sources. The integrated visual analytics methods, allow energy analysts to incorporate their expert knowledge into the analysis, so as to dynamically investigate the observed events and locations, and accurately identify the preferable results. The goal of visual analytics research is to turn the information overload into an opportunity by enabling decision-makers to examine this massive amount of information to make effective decisions.
\end{abstract}

\section{Introduction}

The appraisal of the technically and economically exploitable Renewable Energy Resources (RES) potential can be considered as one of the biggest problems worldwide, with enormous implications for the environment, economy and development for each country. Given a preferred location, energy planning stakeholders must typically evaluate energy demand and aim for the satisfaction of future needs, according to many constraints and factors. The availability of RES at a specific location depends on climate and weather conditions as well as on socioeconomic and landscape criteria and is highly variable. Thus, RES selection and installation, given a variety of exploitation proposals, is a highly complex task that requires the analysis of huge amounts of historic data.

As a consequence, a large number of parameters concerning the implementation of Renewable Energy Technologies must be evaluated by potential investors, in order to assess the technically and economically exploitable potential of RES in a wide geographical area. A comprehensive environmental assessment must consider the following subtopics; (a) a Technical Assessment to identify barriers to 
RES deployment, such as inadequate electricity network infrastructure or natural obstacles, (b) a Land Use Analysis; assessing the changes in land use patterns for setting up RES Wind or solar energy plants, (c) a Social, Ecological and Environmental assessment (e.g. landscape alteration, visual change of rural life or noise) and its impact on the flora and fauna of the area, and (d) a Meteorological Assessment. This is fundamental because the selected assessment actions have to examine and synthesize thoroughly the current state, with respect to technical, landuse, environmental and socio-economic, issues at different levels of intervention. Decision makers must be able to identify a complete framework of performance indices for their planning alternatives. So, a prediction model for these assessment resources should be stochastic in nature to be able to account for the inherent variability. A majority of the existing tools for forecasting these renewable resources give a deterministic forecast, also known as a spot or a point forecast.

From these observations we derive the basic idea and goals of the proposed concept: An integrated service environment for real-time interaction (between human and machine), visualization and decision support by combining the best available data management and data analytics approaches with the best visualization technologies (i.e. innovative visual analytics [1]) over extremely large volumes of heterogeneous data sources. The goal of visual analytics research is to turn the information overload into an opportunity by enabling decision-makers to examine this massive amount of information to make effective decisions. Visual analytics enables people to find hidden relations and to turn the data into useful and defensible knowledge.

The motivation for the proposed framework is the need for energy planners from Greece and Germany to go beyond the analysis of conventional environmental and weather data, from well known databases, satellite images and existing systems, for understanding the relevant patterns in their energy plans within the German and Greek territories. Thus, there is a need for stakeholders to efficiently analyse and visualise an overlay of the historical data (energy, weather, environmental, energy consumption patterns and energy generation data, etc.) that were monitored and measured in real time from existing RES installations in a specific geographical location, along with all data from existing worldwide databases \& tools (PVGIS [2], Poseidon, SoDA [3], etc.). Innovative, stochastic energy assessment modelling and optimization algorithms will be designed as part of services that allow end users to optimize their RES planning strategy through detailed simulations and innovative information visualisation exploration.

\section{The state of the art in this area}

\subsection{Identification and estimation of potential exploitable RES}

Although at the present time they represent a tiny fraction of the world's energy use, their potential is sufficient to completely replace non-renewable sources in the long term. Greece's energy market is at the forefront of transformative changes that are attracting investors from around the globe and especially from Germany where a major energy plan (The Helios project) [4] for the utilization of solar energy in Greece, amounting to 20 billion Euros is under development. Moreover, Wind, or aeolic, power, that offers an alternative that is almost without limit, is driving growth in the renewable energy sector and represents a huge investment potential in Greece. So, with Greece emerging as the energy hub of Southeast Europe, and an aggressive drive for renewable sources to play a major role in the energy supply, the country is at the centre of significant growth opportunities. 
The problem of the identification and estimation of potential exploitable RES in a specific geographical area and in a sustainable manner has been tackled in the last decade with the use of Geographical Information Systems and modelling simulation platforms focusing on numerical weather prediction models, and satellite images. The well known PVGIS (Photovoltaic Geographical Information System) tool [2], provides data (solar radiation data, PV module surface inclination and orientation and shadowing effect of the local terrain features (e.g. when the direct irradiation component is shadowed by the mountains)) for the analysis of the technical, environmental and socio-economic factors of solar PV electricity generation in Europe and Africa and supports systems for solar energy decision-making.

Another tentative tool that is being used by energy planners to address the problem is the SoDa Maps of Irradiation [3], Irradiance, and UV. Similarly, the SolarGIS [5] database and simulation tools provide data calculated using in-house developed algorithms that process satellite imagery, and atmospheric and geographical inputs in different locations. The Centre for Renewable Energy Sources (CRES) has also developed a GIS for assessing the technically and economically exploitable RES potential in Greece [6]. Similar initiatives have been also proposed for wind power exploitation (e.g. WindPro [7]) in Greece as well as in Europe [8]. Within the energy software simulation modelling domain, we can mention the RETscreen [9] tool and HOMER [10] which simulates and compares the performance of multiple energy systems (and can determine the optimal combination of power sources by comparing capital, operating costs and emissions over the life of the system).

Despite lots of interactive GIS and simulation tools and models that are now available to compute the required RES parameters in a given geographical area, the move towards the full exploitation of RES is nevertheless held back by the lack of innovative visualisation and simulation solutions which could improve the delivery of relevant information and end-user experience. With the new solutions the territorial RES potential could be evaluated, even for smaller areas, by enriching the data with big historical data from existing RES installations, so that energy planners could get a truly accurate picture of the long-term risks involved. This would be a fundamental shift from a model-based approach, to a data-driven approach which allows more accurate predictions, but requires services to manage and visualize large amounts of data. Right now there are large volumes of project- or module-level historical data (collected in real time and stored in an anonymised manner) that are being gathered from operating RES installations in Greece and Germany. After careful analysis, these could offer important insights into the true nature of RES operations and maintenance costs, failure rates, and energy generation.

\subsection{Visual Analytics in the area of Energy}

Visual analytics [11, 12] is a novel research field that aims to provide visualisation based methods for human supported analysis of large, complex datasets. Visual analytics differs to classic information visualisation in that it attempts to take into account properties of human cognition, perception and sense making. Essentially, visual analytics aims to develop appropriate visualisation and interaction mechanisms that match the mental processes of humans. Moreover, visual analytics targets applications that present particular challenges, e.g. involve huge volumes of data or complex heterogeneous sources of information that are hard to analyse using automated mining methods. In essence, as it is often mentioned, the overarching vision of visual analytics [12] is to turn the information overload into an opportunity by making our way of processing data transparent for an analytic discourse. 
Ultimately, visual analytics provides technology that combines the strengths of human and electronic data processing, drawing tools from the information visualisation, data mining and cognitive science communities. Visualisation becomes the medium of a semi-automated analytic process, where humans and machines cooperate in a manner which exploits their respective strengths for the most effective results. The user directs the analysis according to his or her specific goals. At the same time, the system provides both an effective means of interaction and data-mining tools for analysis steps, which can be automated.

Visual analytics has a very wide scope and relevant applications have appeared in many different areas. Successful applications have been developed in such diverse fields as environmental monitoring [13], software analytics [14], computer network security [15], electrical grids [16], etc. In general, research in the field of visual analytics has focused on specific applications. Work on general principles, e.g. on evaluation, sense making, perceptual and mental matching, is rather scarce compared to application-specific work.

Considering this, there are elements from applications that involve analysis of spatiotemporal data that could be used in order to benefit the goals of the proposed framework. A good review of the challenges, open issues and most important visual analytics systems that deal with analysis of spatiotemporal data can be found in [17]. In the following, some systems/tools, from which the proposed framework could transfer and further develop existing techniques, are presented.

The Visual analytics Law Enforcement Toolkit (VALET) [18] is a rich interactive environment that supports the exploration and analysis of complex spatiotemporal data. It utilises temporal plots and heatmaps in order to assist law enforcement analysts to detect patterns of criminal behaviour. A tool for powerful analysis of movement data is VisTracks [19]. VisTrack utilizes maps to represent movement data, but at the same time it offers a set of statistical analysis tools to assist in discovering patterns and correlations in data. An overview of issues related to visual analytics for decision support can be found in [20]. Other visual analytics tools for analysis of spatiotemporal data are presented in [21], where different aggregation mechanisms across the time and space dimensions are used in conjunction with heatmap visualisations in order to assist the analyst in detecting important spatiotemporal spots and in [22], where a choropleth map and a parallel coordinate plot allows the user to correlate spatiotemporal data with other sources of information.

\section{The Introduced Framework}

\subsection{The proposed EVRESIS system}

Currently, energy planners and analysts are bombarded with enormous volumes of data that emanate from a variety of sources. So, vital decisions in RES installations can only be achieved through more efficient ways of processing and analysing terabytes of data. EVRESIS introduces a holistic RES assessment framework for future installations of RES assets in specific geographic areas that incorporates traditional well-known assessment models (numerical weather prediction (NWP) models [23], PVGIS data, models from advanced planning tools such as HELIOS 3D, etc.), critical RES operational processes and, consequently, the created micro and macro models that are mapped to appropriate geographic zones.

The main goal of the proposed framework is to tighten the trinity of data management - analytics - visualization through research and development of 
technologies to identify patterns, events and trends in massive amounts of data. This requires, the establishment of sophisticated and technology driven services for decision making and, hypothesis testing, close to the market. EVRESIS presents a unique opportunity for the establishment of a mutually beneficial cooperation scheme between Germany and Greece in the area of energy planning and production based on natural sources.

More specifically, the EVRESIS framework will address the following specific goals:

- To develop a scalable and technology agnostic cloud-based data collection and analysis service infrastructure, capable of dynamically incorporating a large number of heterogeneous input sources (meteorological, multi-sensorial, GIS, etc.) coming from existing RES installations at the global but also at the local level and by analysing them using novel data analytics methodologies. The aim is to build an inclusive and open service infrastructure that can be used in combination with the major global databases and existing information sources.

- To build relevant RES operational profiles (RES micro and macro operational models) for each type of installed RES generation asset in a specific geographic area, in order to match each zone with one and/or more models. The models will consider technical aspects (e.g., installed systems, size/capacity, produced energy, failures, etc.), environmental (weather data, location, GIS data, urban/industrialized area, $\mathrm{CO} 2$ emissions, etc.), economic aspects (e.g., energy costs, installed costs, maintenance costs,), sociological data (e.g. noise, proximity to cultural monuments, etc.) and other short-term time-scaling uncertainties (production risks, climatic uncertainties; temperature, wind speed for wind turbines, solar radiation for solar cells). For the creation of the models we will take into account relevant dynamic and up-to-date data drawn from existing RES installations.

- To establish an Integrated exploitation Assessment RES Model that extends current energy assessment Models and 3D RES software design models (provided by established modeling software like HOMER, RETscreen, SAM [24], PVsyst [25], Helios 3D [26] etc,) that are being used by energy planners and designers by incorporating and integrating the implemented RES parameterized models coming from existing RES installations.

- To implement a Hypothesis-driven Data Investigation for short term and long Term Analysis of future RES installations. A fully-fledged visual analytics framework will offer alternative ways of exploiting large amounts of observed data in order to allow users to visualize and explore how this information changes through time in a spatio-temporal representation of multiple RES micro and macro models (visual, textual, time-series, graphs, vectors, etc) and in multiple visualization environments (Visual Analytics Techniques). The framework will provide extensive functionality to the end-users (planners, designers) and the ability to perform interactive hypothesis testing for evaluating different energy planning rules and options and visually representing the energy performance of different alternatives under real life operational conditions. This module will be open enough in order to be integrated with existing RES monitoring tools, like the insolar product of Inaccess.

- To develop and evaluate a Holistic Modeling and Simulation Framework, based on contingent and robust proactive-reactive energy planning approaches that take into account integrated RES assessment models.

- To create an "open database" of energy assets (instances for RES models from existing RES installations) with corresponding financial, technical, environmental parameters, for the Greek and German territories, that can be 
used for future analysis of RES market growth and RES improvement. Thus, end users may add their own RES installation and energy generation data, and then browse RES data entered by others, or view statistics derived from installation data.

\subsection{The EVRESIS conceptual architecture}

The overall EVRESIS conceptual architecture is graphically depicted in the following figure1.

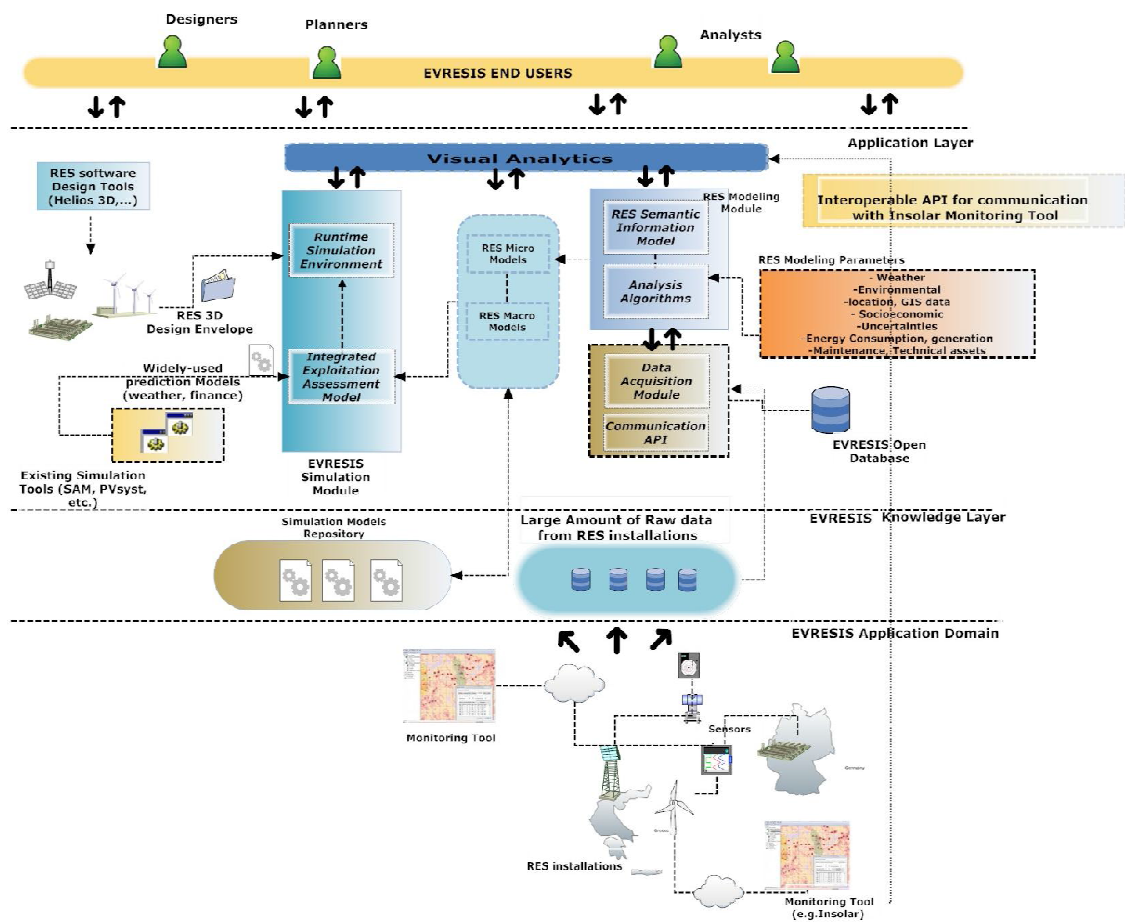

Fig. 1. The EVRESIS conceptual Architecture

The core technological components of the EVRESIS framework are:

- The Data acquisition/collection module, capable of dynamically incorporating a large number of heterogeneous input sources from existing RES installations. A specific API that will be implemented can support the future integration of dynamically updated additional databases of raw data coming from existing RES installations.

- The RES Modeling Module Architecture. The purpose of the RES modeling component will be to provide the necessary tools to access and analyse the data stemming from the data collection module in order to create generalized micro and macro patterns/models as well as specific business process models in a specific geographic area with improved accuracy that do not exist in the existing SoA paradigms. The design of the models will be as abstract as possible in order to enable their easy adaptation to a new plan of RES installation processes.

- The EVRESIS Simulation \& Decision Support Module Architecture. The main purpose of the decision support \& simulation system will be to provide the RES Designers and energy planners, in the early design phases, 
with the missing functionality (in terms of available simulation tools) addressing the simulated energy evaluation (via simulation runs) of the energy performance of RES installation designs prior to their realization, taking into account integrated RES assessment models. In this context, the system will be able to analyze all the incoming heterogeneous and large amount of data and RES assessment models for a focused geographic area. Thus, some of the following functionalities will be supported (a) To give designers the capability to represent via intelligent and innovative visual analytics services the energy performance of RES installations, (b) To provide a direct simulation for a specific location using integrated free maps (e.g. OpenStreet maps) and by executing appropriate integrated RES assessment models during the simulation process along with other existing common models and (c) to provide reliable and accurate energy assessment predictions for concrete RES installations that take into account multiple criteria (technical, operational, performance, environmental, socioeconomic, etc.) and dynamic activities within a preferable environment (e.g. uncertainties, transitions between geographic zones, etc) and (d) To provide tools and methods for interoperability with existing energy simulation tools and engines. A specific interoperable module will be able to involve the development of separate toolkits for seamless execution of the EVRESIS time-varying simulation data within the context of other existing simulation tools.

- The EVRESIS Visual Analytics framework (figure 2) which will comprise the main user interface for all EVRESIS end-user groups. It will provide visualization and interaction mechanisms to the analysts /modelers to abstract, query, retrieve, synthesize and understand the large amount of raw data and models extracted from the Data collection and analysis platform, in order to automatically detect spatio-temporal correlations of monitored parameters as well as for hypothesis testing and evaluation of different RES installation alternatives. Thereby, advanced visual interfaces will empower the users to interactively explore patterns in large-scale data sets by crosslinking the formerly disjoint data sources in a Visual Analytics Framework.

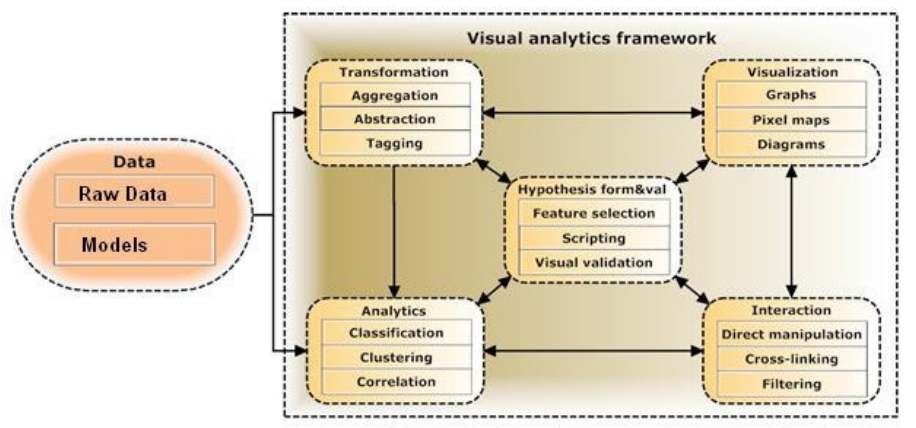

Fig. 2. The EVRESIS Visual Analytics Framework

The proposed system targets some the following user groups:

- Governmental authorities (cities, regions) to assess the economic and environmental impacts of RES planning scenarios, to optimise their local energy strategy to cost-effectively reduce $\mathrm{CO} 2$ emissions, to develop new energy plans in 
and/or identifying the most attractive and exploitable areas in their regions for RES planning

- Private companies and industries involved in the energy sector and looking for a new business opportunity in RES, either as constructors or as consultants for such RES installations. The provided location analysis visualisation process can help them to identify alternative RES assets and examining candidate locations that are deemed to have fewer obstacles to the RES potential projects and that are more profitable at the initial stages of the development.

- RES installers and manufactures that have already installed their infrastructure in the Greek and German territories. The system will enable them to better design and configure their infrastructure that will result in upgraded products and services to their customers as well as to decreased operational and infrastructural costs and complexity.

- The general public and the individual citizens, who can have access to RES data entered by others with similar needs and capabilities, in order to compare data and identify the potential of RES within their location as well as the derived energy profit.

\section{Conclusion and Planned Activities}

In Berlin, on March 27 2013, the State Secretary at the Federal Environment Ministry in Germany signed a joint declaration of intent with the Greek Ministry of the Environment, Energy and Climate Change (YPEKA) and the EU Commission's Task Force for Greece on strengthening cooperation in the field of renewable energies. This entails the future utilization of a platform like the proposed one to support integrated services and tools for the public authorities, the wind energy sector, the solar energy sector and finally citizens in both countries to help them in their Sustainable Energy Action Plans. So the present paper proposed an assessment tool for visualizing and exploring appropriate assets for supporting the current decisions of energy planners and analysts. Next steps include to apply this framework into a realRES case, in order demonstrate its analytical potential.

\section{Acknowledgement}

This work was partially supported by the a) EU FP7/2007-2013, under grant agreement no. 288094 (project eCOMPASS), b) the National programme PAVET 2013, project EnergyVisualAnalytics, from the General Secretariat for Research and Technology and c) the FP7 project NEMESYS, under grant agreement no. 317888.

\section{References}

1. Visual Analytics for Large amount of data, http://en.wikipedia.org/wiki/Visual_analytics, accessed at April 2014

2. European Photovoltaic Geographical Information System (PVGIS), http://re.jrc.ec.europa.eu/pvgis/apps4/pvest.php?lang=en\&map=europe, accessed at April 2014

3. Solar Energy for professionals, Soda Maps of Irradiation, Irradiance, and UV: http://www.soda-is.com/eng/map/maps_for_free.htmlBruce, accessed at April 2014

4. The Greece-Germany Helios project, http://www.project-helios.gr, accessed at April 2014

5. Photovoltaic Softwares for simulation and decision support, http://photovoltaicsoftware.com/solar-irradiation-database.php, accessed at April 2014

6. GIS for assessing the technically and economically exploitable RES potential in Greece, http://www.cres.gr/kape/projects_26_uk.htm, accessed at April 2014

7. WindPro energy simulation and decision support Tool, http://www.emd.dk/windpro/frontpage, accessed at April 2014

8. Centre for Renewable Energy Sources (CRES) European projects for energy: http://www.cres.gr/kape/pdf/download/Black_Sea.pdf, accessed at April 2014 
9. The RETScreen Clean Energy Project Analysis Software, http://en.wikipedia.org/wiki/RETScreen, accessed at April 2014

10. HOMER- Energy Modeling Software for Hybrid Renewable Energy Systems, http://homerenergy.com/, accessed at April 2014

11. Daniel Keim, Gennady Andrienko, Jean-Daniel Fekete, Carsten Görg, Jörn Kohlhammer and Guy Melançon. Visual Analytics: Definition, Process, and Challenges. Information Visualization, Lecture Notes in Computer Science, 2008, Volume 4950/2008, 154-175.

12 J.J. Thomas and K.A. Cook, Illuminating the Path: The Research and Development Agenda for Visual Analytics. National Visualisation and Analytics Centre. 2005.

13. Ulanbek Turdukulov, Connie Blok. Visual analytics to explore iceberg movement. Workshop on GeoVisualization of Dynamics, Movement and Change at the AGILE 2008 conference.

14. Dennie Reniers, Lucian Voinea, Ozan Ersoy, and Alexandru Telea. A Visual Analytics Toolset for Program Structure, Metrics, and Evolution Comprehension. In Proceedings of the 3rd Intl. Workshop on Academic Software Development Tools and Techniques (WASDeTT-3). 2010

15. Florian Mansmann, Daniel A. Keim, Stephen C. North, Brian Rexroad, Daniel Sheleheda. Visual Analysis of Network Traffic for Resource Planning, Interactive Monitoring, and Interpretation of Security Threats. IEEE Transactions on Visualization and Computer Graphics. Vol. 13. No. 6. 2007

16. P.C. Wong, K. Schneider, P. Mackey, H. Foote, G. Chin, R. Guttromson, J. Thomas. A Novel Visualization Technique for Electric Power Grid Analytics. IEEE Transactions on Visualization and Computer Graphics 15, 3 (2009), 410-423

17. Gennady Andrienko et al. Space, Time and Visual Analytics. International Journal of Geographical Information Science, Volume 24 Issue 10 2010, Pages 1577 - 1600: Geospatial Visual Analytics: Focus on Time Special Issue of the ICA Commission on GeoVisualization

18. Abish Malik, Ross Maciejewski, Timothy F. Collins, and David S. Ebert. Visual Analytics Law Enforcement Technology. IEEE International Conference on Technologies for Homeland Security, 2010.

19. Stephen G. Eick. Geospatial visualization with VisTracks. Wiley Interdisciplinary Reviews: Computational Statistics. Volume 2, Issue 3, pages 272-286, May/June 2010.

20. Jörn Kohlhammer, Thorsten May and Marcus Hoffmann. Visual Analytics for the Strategic Decision Making Process. NATO Science for Peace and Security Series C: Environmental Security, 2009, Part 5, 299-310.

21. Abish Malik, Ross Maciejewski, Timothy F. Collins, and David S. Ebert. Visual Analytics Law Enforcement Technology. IEEE International Conference on Technologies for Homeland Security, 2010.

22. Jern M, Thygesen L, Brezzi M. A web-enabled Geovisual Analytics tool applied to OECD Regional Data, Proceedings of Eurographics 2009, Munchen, March 2009.

23. Numerical_weather_prediction models, https://en.wikipedia.org/wiki/Numerical_weather_prediction, accessed at April 2014

24. The System Advisor Model (SAM) to facilitate decision making for people involved in the renewable energy industry: https://sam.nrel.gov/, accessed at April 2014

25. PVsys Photovoltaic software: http://www.pvsyst.com/en/software, accessed at

April 2014 26.HELIOS 3D powerfultool for utility scale solar plants:

http://www.helios3d.com/index.php/helios-3d.html, accessed at April 2014 